Nahla HUSSEIN

\title{
ÜBERLEBENSKÜNSTE UND INTEGRATIONSVERSUCHE IN DER DEUTSCHSPRACHIGEN LITERATUR MITTEL- UND OSTEUROPAS
}

Bis 1939 galt die Region Mittel- und Osteuropa als Region der großen ethnischen Vielfalt auf kleinem Raum, doch erst nach dem Fall des eisernen Vorhangs 1989 konnte die Rede von der multikulturellen Geschichte dieser Region laut werden. In der Literatur dieses Gebiets kommt das nun klar zum Ausdruck. Deshalb zählen diese beiden Jahrzahlen (1939 und 1989) zu den bestimmenden Eckdaten in der Historie Zentraleuropas. Wie so viele Regionen der Welt in dieser Zeit musste Zentraleuropa im 20. Jahrhundert durch entscheidende Veränderungen der Machtverhältnisse hindurch. Mitte des 20. Jahrhunderts begann in Ungarn die Zeit großer Umwälzungen und Bürgerkriege. In den 1980er Jahren zerfiel der Vielvölkerstaat mit dem damaligen Namen Jugoslawien. Nach 1989 durften zumindest offiziell die Einwohner des ursprünglichen Ostblocks in die meisten Länder reisen, was ihnen vorher verwehrt war. Somit musste die Bevölkerung in den multikulturell geprägten und transkulturell umkämpften Gebieten Zentraleuropas große Verluste erleiden, die sich nicht nur auf das Materielle beschränkten, sondern weitaus tiefer in das Zusammenleben in die Gesellschaft und in die individuellen Überzeugungen eingriffen. Auch wenn einige vielleicht äußerlich unversehert fliehen konnten, begann nach dieser Flucht die Auseinandersetzung mit dem Vergangenen und eine Evaluation, so dass die Identitätsfrage hier oftmals Thema wurde. Der Kommunismus, „,[d]as Konzept einer universalen Emanzipation [dessen] erstes Ziel eine bessere Welt ist, erst danach die Prosperität seiner lokalen Gemeinschaft oder das Interesse seiner partikularen - ethnischen, religiösen oder Gender - Identität", ${ }^{1}$ so Daniela Finzi, erwies sich in der Praxis als eine schmerzhafte Enttäuschung.

Finzi, Daniela 2013: Saša Stanišić: Wie der Soldat das Grammofon repariert. In: Unterwegs zum Andern? Literarische Er-Fahrungen der kriegerischen Auflösung Jugoslawiens aus deutschsprachiger Perspektive. Tübingen: francke Verlag. S. 235 - 252, hier: S. 237. 
Hierzu entwickelt Brigid Haines im Jahr 2008 den Fachbegriff Eastern Turn als Benennung für die Literatur dieser Region in der nachkommunistischen Phase, denn diese Literatur „weist in individuellen Texten eine Neubewertung von Identitätsfragen auf, sowie eine thematisch vereinigende Meta-Erzählung des Post-Kommunismus. “2 Dieser Beitrag zielt darauf $a b$, anhand der Werke dreier Schriftsteller aus Ungarn und dem ehemaligen Jugoslawien die Versuche der Protagonisten, ihre Vergangenheit zu verarbeiten und sich mit der Realität auseinanderzusetzen bzw. in ihr eine neue Heimat zu schöpfen, darzustellen. Dabei wird das Besondere im Werk dieser Dichter hervorgehoben. Um eine theoretische Variante des Zusammenlebens von Menschen verschiedener Ethnien im Zuge der voranschreitenden Globalisierung und jenseits der ursprünglichen Ost-WestAufteilung anzubieten, entwickelte Wolfgang Welsch bereits im Jahr 1995 die Theorie der Transkulturalität, die der Theorie der Interkulturalität gegenüber laut Welsch - den Vorteil habe, dass ihr Kern darin liege, dass sie keinen Menschen als einen Anderen identifiziert, der aus dessen Perspektive anders sei, sondern der einer kompletten Menschheit gegenüber offen sei, weil nicht zuletzt jeder Mensch ein Stück vom Eigenen vom Anderen schon enthalte. Die Theorie der Transkulturalität sehe die Kulturen nicht als Kugeln, sondern erkenne die Grenzen zwischen den Kulturen als flexibel und erlaube deshalb eine gegenseitige Bereicherung. Das Konzept der Multikulturalität beruht zwar auch auf der Vorstellung von Kulturen als Kugeln, wäre aber dem neuen Zustand einer zusammenwachsenden Welt gerade aus diesem Grund nicht gewachsen. Anhand ausgewählter Werke von drei ursprünglich osteuropäischen Autoren ist in diesem Beitrag die Effizienz der Theorie der Transkulturalität zu untersuchen und sind ihre Grenzen zu erkennen.

Aktuell veröffentlichten mehrere Autoren, die Ende der 1960er oder in den 1970er Jahren geboren sind und ursprünglich aus Zentraleuropa abstammen, jedoch seit zwei oder drei Jahrzehnten in Deutschland leben, ihre Bücher im deutschsprachigen Raum. Sie fühlen sich in der deutschen Sprache zu Hause und sie schreiben in eben dieser Sprache. Diese neue Literatur wird

Rock, Lene 2012: Überflüssige Anführungsstriche: Grenzen der Sprache in Terézia Moras Alle Tage \& Saša Stanišić’ Wie der Soldat das Grammophon repariert. In: Germanica 51. S. 48. 
zum Teil widersprüchlich aufgenommen. Gerade wegen der inhaltlichen wie stilistischen Fülle dieser Dichtung und als ein Zeichen gelungener Integration wird seit Mitte der 1980er der Adelbert-von-Chamisso-Literaturpreis vergeben. ${ }^{3}$

Die Deutschen haben mir ihre Muttersprache anvertraut, und ich habe in ihrer so wunderbar gefügige und gepflegte Sprache Erkenntnisse meines nomadischen Muttervolkes eingepackt, es mit schamanischem Geist und dichterischer Seele angehaucht und den Spendern eines neuen Kommunikationsmittels als Dank zurückgereicht, ${ }^{4}$

so der deutschsprachige Migrantenschfriftsteller Galsan Tschinag, aus der Mongolei. Inzwischen wird die Kritik laut, dass solche Autoren einen Büchermarktbedarf nach dem Neuen oder Extremen abdecken und es wird zuletzt sogar konstatiert, wie überflüssig sich dieser Preis gemacht habe. ${ }^{5}$ Dieser Vorwurf lässt sich vielleicht nicht für alle Schriftsteller belegen, doch selbst der Chamisso-Preisträger aus dem Jahr 2008 Saša Stanišić steht den Voraussetzungen des Preises kritisch gegenüber, denn für ihn ist die Sprache des Dichtens an sich eine Fremdsprache, die jedes Mal neugelernt werden müsse. ${ }^{6}$ Andere Autoren sehen in den Bezeichnungen der aktuellen deutschsprachigen Literatur Zentraleuropas - als Migranten-, Gastarbeiter-, oder Ausländerliteratur ${ }^{7}$ naive Kategorisierungen mit dem Ziel der Identifizierung oder der Reduzierung, die die Bedeutung der Literatur als grenzenlose Kreativität und Spiel der Referenzialität schlechthin nicht begreift. In der erwähnten Dichtung geht es - wie es hier noch zu beweisen ist - um die Frage der Identität und der Neuverortung des Einzelnen. Die Protagonisten solcher Werke erpoben wiederholt neue Wege, sich selbst zu

Ebda., S. 48.

Kimmich, Dorothee 2012: Lob des »Nebeneinander«. Zur Kritik kulturalistischer Mythen bei Kafka und Wittgenstein. Darunter mehrere Aufsätze von den Autoren. In: Dorothee Kimmich $(\mathrm{Hg})$; Sigrid Karin Amos: Kulturen in Bewegung. Beiträge zur Theorie und Praxis der Transkulturalität. Bielefeld: transcript. Hier: S. 66.

5 Fisch 2015: »Es erkenne mich die Welt, auf dass sie mir verzeihe« Aufsätze zu Adelbert von Chamisso (1781-1838), Paul Ernst (1866-1933) und Hubert Fichte (1935-1986). Berlin: Weidler. (Beiträge zur transkulturellen Wissenschaft. Band 1.), S. 11-12.

$6 \quad$ Rock: op.cit. S. 55.

Ebd., S. 56. 
finden, Konflikte zu meiden und Kontakte mit der Gesellschaft zu knüpfen. Aufgrund der Fülle an unverarbeiteten Ereignissen und Emotionen verläuft das Erzählen und das Erinnern dieser doch nahen Vergangenheit nicht immer konsequent. Von den Protagonisten der Werke ist zudem ihr Wahrheitsanspruch anzuzweifeln. Es ließe sich nicht zuletzt postulieren, die Wahrheit gelte gar nicht als Kriterium der Aufarbeitung einer Vergangenheit, viel mehr sei es die Fähigkeit der Figuren das Vergangene überhaupt und irgendwie wahrzunehmen.

Daniela Finzi ${ }^{8}$ meint hierzu, dass das Erinnern und auch das NichtErinnern die Zukunft des südosteuropäischen Raums folgenschwer bestimmen wird. Begründet wird diese These durch Aleida Assman Theorie (1999), ${ }^{9}$ die den entscheidenden Wert solcher Texte darin sieht, dass sie sozusagen eine Phase der menschlichen Geschichte bestimmen, indem sie den Abfall des Erinnerungsprozesses nicht verloren gehen lassen. Im vorliegenden Beitrag geht es um drei Autoren, nämlich Zsuzsa Bánk aus Ungarn (1965 geboren), Saša Stanišić (1978) und Marica Bodrožić (1973) beide aus dem ehemaligen Jugoslawien. Indem sie auf Deutsch schreiben, wird das Eigene verfremdet und aus einer anderen Perspektive betrachtet. Wie noch darzustellen ist, unternehmen die Protagonisten in diesen Werken mit zerrißendem Schmerz immer wieder den Versuch, ihre Identität in einer Gegenwart zu etablieren, doch kommen sie ohne die lange noch nicht verarbeiteten Vergangenheit mit sich selbst nicht ins Reine. Hierzu spricht der polnische Autor und Literaturkritiker Andrschj Stasiuk im Jahr 2008 vom „Spannungsverhältnis zwischen den homogenisierenden und den differenzierenden Tendenzen “10. Bei den Versuchen der Aufzeichnung der eigenen Identität zeigt jede(r) der gewählten AutorInnen einen eigenen Schreibstil auf, der sozusagen auf Wurzeln in der osteuropäischen Tradition verweist.

\footnotetext{
$8 \quad$ Finzi: op.cit. S. 238.

9 Assmann, Aleida 2009: „Unbewältigte Erbschaften. Fakten und Fiktionen im zeitgenössischen Familienroman“, in : A. Kraft / M. Weißhaupt (Hrsg.), Generationen. Erfahrung - Erzählung - Identität, UVK. S. 49-69. S. 68.

Makarska, Renata 2012: Kakanien der neuen Generation Zentraleuropa zwischen Transkulturalität und Differenz. In: Dorothee Kimmich (Hg); Sigrid Karin Amos: Kulturen in Bewegung. Beiträge zur Theorie und Praxis der Transkulturalität. Bielefeld: transcript. S. 235 - 260, hier: S. 251.
} 
In den hier von mir ausgewählten zwei Familienromanen der Autorin Zsuzsa Bánk sind zwei Generationen darum bemüht, aus der Gegenwart einen lebbaren Raum zu machen. „Der Schwimmer“ (2002) zeigt die Reaktionen einer Familie auf die Revolution von 1956 in Ungarn. Während sich die hartarbeitende Mutter mit der Realität nicht abfinden kann, das vom Bürgerkrieg gebeutelten Ungarn hinter sich lässt, schlagartig und wortlos vom "gottverdammten, verlassenen Osten" (Sch.," 27 ) in den Westen flieht und dadurch die Familie in Verruf bringt, ist der überforderte und wahrscheinlich deshalb einsilbige Vater bemüht, den Kindern als ein Vobild zu dienen. Bei diesem endlosen Warten nehmen die Kinder alle Naturveränderungen in der Umgebung als Zeichen der baldigen Rückkehr der Mutter wahr. Hier steht „das Schwimmen“ für die Bemühungen der Kinder um ein Eintauchen in andere Wirklichkeiten, das eigentlich nicht weiterhilft. Trotz allem gesteht Kata, die Tochter, Ich-Erzählerin und Hauptfigur im Roman: „Es gibt Zeiten, die ich ertrage, andere nicht" (Sch., 8). Der Roman hinterlässt sozusagen Katas Nachricht, die besagt, ,[w]ir sind allein gelassene Kinder, fremd und unbehaust im Universum". ${ }^{12}$

Im zweiten Roman Zsuzsa Bánks „Die hellen Tage“ (2011) lässt sich eine Art Fortsetzung zu dem ersten Roman erkennen. Darin sind die Protagonisten Immigranten aus Ungarn und ihre Kinder werden als zweite Immigrantengeneration dargestellt. Es handelt sich um die Freundschaft zwischen den Kindern Aya, Seri und Karl in Deutschland, die in ihrer Kindheit Verluste erleiden müssen, und durch die anhaltende Unterstützung ihrer Mütter lernen, die ausgelösten Erschütterungen zu überstehen. Im Laufe ihrer Studienjahre in Rom gehen sie durch Höhen und Tiefen des Lebens, sie üben Widerstand aus und werden daran zu Erwachsenen. Auf der Zunge eins der Kinder, Seris, werden die Ereignisse in Form eines Monologs mit detailierten Natur- und Gefühlsbeschreibungen erzählt. Seri bewundert durchgehend die ungarische Familie. Die Hauptfigur im Roman ist eine der Mütter mit Namen Évi. Trotz ihres prekären Lebens als Analphabetin und ungarische Flüchtilinge, steht sie anderen bereitwillig zur Verfügung. Sie weiß

11 Sch. wird als Abkürzung für den Roman „Der Schwimmer“ benutzt.

12 Truchlar, Leo 2006: Zsuzsa Bánk. Der Schwimmer. In: Schwelle. Passage. Verwandlung. Ein Interpretationsentwurf. Wien: Lit Verlag GmbH. \& Co. KG. S. 335 - 337, hier: S. 336. 
das Schöne im Leben zu empfinden und Schicksalsschläge zu überleben. Ihre Lebenskunst fasst sie zusammen: „Die hellen Tage behalte ich, die dunklen gebe ich dem Schicksal zurück“" (Ta., ${ }^{13}$ 515). Als ihre Tochter, Aja, Ärtzin wird, fallen die Grenzen zwischen Osten und Westen, aber Aja - Seris Vorbild - meint, für sie kann Deutschland keinen „Zwischenhalt“ mehr sein (Ta., 540). Das Werk ist somit ein Plädoyer auf das Leben im Einklang und ein Bezug zur Mutterliebe. Zsuzsa Bánk zeigt große Gewandtheit bei den Gefühlsbeschreibungen. Während es ihr in „Der Schwimmer“ ein detailliertes Bild des Leidens einer zersplitterten ungarischen Familie darzustellen gelingt, zeigt sie auf der anderen Seite in „Die hellen Tage“ meisterhaft die einfältige Lebenskünstlerin und ungarische Flüchtlinge Évi, die sich anzupassen und spontan positive Energie zu schöpfen weiß, und gerade deswegen anderen Menschen Beistand leistet.

Wenn man hier die Theorie der Transkulturalität einbezieht, kann man in den zwei Werken unterschiedliche Einstellungen der sich verändernden Welt gegenüber erkennen. Die Protagonisten im zweiten Roman „Die hellen Tage“, die selbstverständlich auf einer weiteren Stufe der Verflechtung mit der neuen Gesellschaft sind, sind stärker, offener und flexibler, und die heranwachsenden Kinder entwickeln sich und sind ebenfalls ein gutes Beispiel für den heutigen „transkulturellen“ Menschen, für den die Verfelchtung mit der Gesellschaft eine angemessene Herausforderung ist, an der er sich entfaltet. Jedoch erfährt man gegen den Schluss des Romans „Die hellen Tage“, wenn Aya Ereignisse zu hinterfragen beginnt, dass der Mann, der die ungarische Familie ab und zu besuchen kommt, Ayas Vater ist, und dass ihre leibliche Mutter unbekümmert woanders lebt. Somit steht das Harmonische im ganzen Werk in Frage. Außerdem wird Zsuzsa Bánks Werk kritisiert; während dem ersten Roman „Der Schwimmer“ Eintönigket, Schwermut und Weltverlorenheit vorgeworfen wurde, sah die Literaturkritik im zweiten „Die hellen Tage" eine mythisch überhöhte, spannungslose Geschichte, und nichts Anderes als eine Allegorie auf die unstillbare Sehnsucht nach vergangenem Glück. Es bleibt hinzuzufügen, dass in beiden Werken die Mutter die entscheidende Rolle bei der Bestimmung, wo denn eine eigentliche Heimat überhaupt sei, spielt. Die beiden Texte zeigen die unstillbare Sehnsucht nach

Ta. wird als Abkürzung für den Roman verwendet. 
Geborgenheit in der Figur der Mutter und der Mutternähe an sich. Allerdings fehlt jedewede Konfrontation mit der Realität, zudem sind die Hauptfiguren und Erzähler in beiden Werken emotionale Frauenfiguren. Männerfiguren sind dabei einsilbig oder bleiben nur eine gute Erinngerung, was ebenfalls die Mangel des Rationalen symbolisiert. Hieraus ist zu schlussfolgern, dass es in beiden Romanen nur um scheinbare Verflechtungsversuche geht. Transkulturalität ist in diesem Sinn hier also nicht erreichbar.

Eine andere Herangehensweise mit der Verflechtungsfrage stellt Saša Stanišić in seinem Roman „Wie der Soldat das Grammofon repariert“" (2006) dar. Stanišić wendet inhaltlich wie strukturell besondere und eigenartige Kunstgriffe an, um die sonderliche Geschichte der Heimat des Protagonisten $\mathrm{zu}$ durchleuchten, um seine befremdliche Lebensgeschichte erforschen und seine sonderbare Identität ergründen zu können. Trotz des trickreichen und geschickten Vorgehens erspürt die Hauprfigur, die zudem als Ich-Erzähler aggiert, zuletzt der wiederholten Bemühungen überdrüssig, dass es einzig und allein um das Überleben geht. Nach wie vor lässt sich weder die Geschichte der Heimat in klaren Zügen erzählen, noch ist die eigene Historie auffindig zu machen. Von einer klaren Identität ist hier ganz $\mathrm{zu}$ schweigen. Den Erstlingsroman von Saša Stanišić wollte die Literaturkritik in semibiographischen Zügen bannen. Dieser Auffassung liegt die Annahme zugrunde, dass Schriftsteller in ihrem Kunstwerk in ihre Heimatländer zurückkehren, um sie in der Erinnerung zu verklären. Stanišić wehrt sich dagegen, ,[...] sobald soziopolitische und biographische Faktoren zur Deutung und Beurteilung eines künstlerischen Werkes fungieren, wird ein ästhetischer Diskurs verhindert, zumindest behindert." ${ }^{14}$ Nach dem Zerfall Jugoslawiens und der Flucht in die Bundesrepublik Deutschland erweist sich die Fabulierlust als lebenswichtig für den Protagonisten im Roman mit Namen Alks. Denn diese verhilft ihm, die Vergangenheit zu verzaubern, um für sich einen Platz in der Gegenwart zu finden. Aleks nimmt sich vor, immer weiter zu erzählen. Er ist für das „Undsoweiter!“ (Wie, 23) ${ }^{\text {“15 }}$ und wird zum

14 Maćek, Amalija 2008: Balkanbilder bei Saša Stanišić und Catalin Dorian Florescu. In: Slavija Kabić, Goran Lovrić (Hg.): Mobilität und Kontakt. Deutsche Sprache, Literatur und Kultur in ihrer Beziehung zum südeuropäischen Raum. Kroatien. S. 348. 
„Künstler des guten Unvollendeten“ (Wie, 24). In der statischen Kunst sind Aleks Gemälden die Zusammenfassung seiner Befürchtungen und der im Leben unerreichten Wünsche: Die Trauer um seine Heimat ist im Gemälde „Menschenschatten unter einer Laterne ohne Mensch“ (Wie, 24) zu erkennen. Hervorstechend wird gleich bei der Lektüre, dass Saša Stanišić anfangs lange, detaillierte Überschriften zum Einsatz bringt, die als Zusammenfassung des jeweiligen Kapitels gelten können, und komplexe, anenandergereihte Konstruktionen verwendet. Außerdem bringt er sehr viele, aufeinanderfolgende und kreative Metapher zur Anwendung, die auf den ersten Blick rätselhaft wirken und manchmal zum Barockenhaften tendieren. Der Roman ist aus Collagen zusammengesetzt, weist mehrere Binnengeschitchten sowie eine Formen- und Genrevielfalt auf. Darüber hinaus wechseln sich noch die Erzählperspektiven. Durchgehend kommt es auf den Leser an, viele Zusammenhänge und Übergänge selbst zu erarbeiten, wobei er $a b$ und zu an der Glaubwürdigkeit der Erzähler zweifeln muss. Hierzu meint Bühler-Dietrich, ${ }^{16}$ bei der Aufarbeitung der Erfahrungen von Verlusten stehe nicht die Frage nach der Rekonstruktion der Wahrheit im Zentrum der Texte, sondern der Prozess der Ablösung und der transformativen Rekonstruktion des Subjekts. Aleida Assman klassefiziert solcher Erzählstrukturen als Strukturen des gegenwärtigen Generationenromans.

Im Gegensatz zur epischen Familiensaga ist im gegenwärtigen Familienroman die Erzählstruktur mehrfach gebrochen. An die Stelle eines glatten Erzählflusses sind neue Formen der Montage, an die Stelle eines mit privilegierten Wissen und Autorität ausgestatteten Erzählers ist der stockende Rhythmus der Erinnerung getreten. Erinnerung, so darf man zusammenfassen, ist die Muse des neuen Generationenromans. ${ }^{17}$

Stanišićs Werk lässt sich in die drei Lebensabschnitte des Protagonisten aufteilen. In jeder Lebensperiode setzt er je nach Alter und Abstand zum Geschehen andere Strategien und Erzähltechniken der Aufarbeitung der Vergangenheit ein. Auf solche Textmanöver greift er zurück, da die Erinnerung lückenhaft ist, und um eben den Erzählfluss nicht abbrechen zu

$16 \quad$ Vgl.: Bühler-Dietrich Ebda., S. 42.

17 Aleida Assmann 2009: „Unbewältigte Erbschaften. Fakten und Fiktionen im zeitgenössischen Familienroman“, in : A. Kraft / M. Weißhaupt (Hrsg.), Generationen : Erfahrung - Erzählung - Identität, UVK. S. 53. 
lassen. Der Sprache gegenüber ist er ohnehin skeptisch. Rock beurteilt dieses Unterfangen damit, dass gerade traumatische Erfahrungen, und der Versuch, sich von der Kindheit zu verabschieden, interessante erzähltechnische Aspekten der Narrativität des Gedächtnisses verursachen. Besonders interssant wird es, wenn viele Leerstellen vorkommen. Das ist eigentlich die treibende Kraft des Erzählens. ${ }^{18}$ In der ersten Lebensphase wird der serbisch-bosinsche Protagonist Aleksander aus der umkämpften Stadt Višegrad porträtiert. Dieser Teil besteht aus vielen Anekdoten, Idealisierungen und Demoniserungen. Von seinem Großvater erbt Aleks neben der Zauber- und der Fabulierkunst die Loyalität zum Kommunismus. Diesbezüglich meint Aleks, „Wenn man Titos Bild von der Klasse wegnimmt, käme anstelle dessen nur Omas Gobelins Schiff im Sturm " (Wie, 8). Alles wird wie in einer Spirale, detaillversessen und wiederholt beschreiben, womit der Ich-Erzähler den Schein erweckt, alles möglichst realitätsnah geschildert zu haben. Trotzdem sind keine Erkenntnisse zu erreichen. Aleks bleibt nur Sammler und erreicht den Status eines Interpreten nicht. Im ersten Werkteil gestaltet er sozusagen einen „Wald aus erfundenen Wahrheiten. ${ }^{19}$ Die Überschriften enthalten ironischerweise vereinzelte und zusammenhanglose Wahrheiten neben Halbwahrheiten. „Wie lange ein Herzstillstand für Hundert Meter braucht, wie schwer ein Spinnenleben wiegt, warum mein Trauriger an den grausamen Fluss schreibt und was der Chefgenosse des Unfertigen als Zauberer draufhat" (Wie, 11). Im zweiten Lebensabschnitt ist Aleks in Deutschland und erlebt die Vorfälle nicht mehr unmittelbar. Zehn Jahre lang erfährt er mittels Briefen und Telefonaten eines Freundes von den Ereignissen in Jugoslawien. Der Freund kommentiert die Vorkommnisse, , ,...] als hätten wir nur das gelernt in all den Jahren der Nachbarschaft und der Brüderlichkeit und der Einheit [...]. Mein Hass ist endlos, Aleksander. Auch wenn ich die Augen schließe, ist alles da“" (Wie., 145). In diesem Teil gibt Aleks das Erinnern auf, denkt nicht mehr und ist verzweifelt, weil er Identität mit Alterität nicht in Einklang bringen kann, er sieht in sich ein „Gemisch ein Halbhalb“ (Wie, 53), mit widerstreitenden Wesenszügen. Bevor Alex in der dritten Phase in die Heimat zurückfährt,

\footnotetext{
$18 \quad$ Vgl.: Rock S. 51.

19 Bühler-Dietrich, Annette 2012: Verlusterfahrungen in den Romanen von Melinda Nadj Abonji und Saša Stanišić. In: Germanica 51. S. 41.
} 
muss er über das Lückenhafte in seinem Gedächtnis recherchieren, weil er vieles verdrängt hat. Das Erinnern plagt ihn. Dazu sagt der Protagonist: „[Ich will mich] rasieren, der Bart ist mir voller Erinnerung an die schlimmste Nacht. [...] Wie schwer wiegen Erinnerungen in einem Bart?“" (Wie., 218f.). Zwar ist die Erinnerung identitätsstiftend, aber auch zugleich identitätszerreisend. Entsprechend Rock sei man beim Erinnern dazu verpflichtet, zwischen wahren und richtigen Aussagen zu unterscheiden. ${ }^{20}$

Obwohl Aleks durch seine Erzählkunst die Welt mit neuen Farben färbt, das Freund-Feind-Schemata außer Kraft setzt, ${ }^{21}$ den Schrecken mittels der Sprache verzaubert, ${ }^{22}$ vom Realismo Magico beeinflusst sureale Züge verwendet und vom Orientalismus geprägt idealisiert und demonisiert, so Amalija Maćek im Jahr 2009, ${ }^{23}$ findet er ausschließlich in der Erinnerung eine gemeinsame Sprache mit den Zurückgebliebenen und kann sich mit dem Nachkriegsbosnien trotz allem nicht abfinden. Zum Schluss des Romans muss Aleks traurig zugeben, dass ihm viele Erkenntnisse über das Menschliche fehlen und dass seine Erinnerungen amputiert sind. Laut Freud sind Melancholie, Trauer und Nostalgie komplezierte Trennungsprozesse. ${ }^{24} \mathrm{Er}$ stirbt, weil er sich mit der Vergangenheit nicht versöhnen kann, in einer allegorischen Szene am Grab seines Großvaters, und zieht währenddessen einen Vergleich zwischen sich und dem jungen, dreisten Fluss Drina (Wie, 313). Somit schließt der Roman mit dem Fluss als eine Art Metapher ab, einerseits als Zeuge für die Vergangenheit, andererseits aber auch als Hoffnung auf die Zukunft. Bei Saša Stanišićs scheitern jedewede Versuche der Verflechtung mit den Wurzeln, nicht einmal mit der transkulturellen Heimat kommt er zurecht. Über die Integration in die Wahlheimat Deutschland äußert er sich mit keinem Wort.

\footnotetext{
$20 \quad$ Vgl., Rock, Ebd., S. 55.

$21 \quad$ Finzi: op.cit. S. 245.

22 Vgl., Thuswaldner, Anton 2006: Die neuen Namen, in: Volltexte 28 (6). S. 5 - 7, hier: 5.

23 Vgl.: Maćek, Amalija 2008: Balkanbilder bei Saša Stanišić und Catalin Dorian Florescu. In: Slavija Kabić, Goran Lovrić (Hg.): Mobilität und Kontakt. Deutsche Sprache, Literatur und Kultur in ihrer Beziehung zum südeuropäischen Raum. Kroatien: Verlag. S. 347 - 354. Abonji und Saša Stanišić. In: Germanica 51. S. 35 - 46, hier: S. 36.
} 
In ihrem Werk geht es Marica Bodrožić um das Ergründen der eigenen Wurzeln, das Öffnen auf den Anderen und die ständige Entwicklung des Selbst, das sich in erster Linie in der literararischen Sprache ereignet. Ist der dominierende Faktor in einem Werk das grenzenlose Gefühl, so ist im nächsten der grenzenlose Verstand als ein Weg zur erwünschten Erkenntnis. Der Mensch ist für diese Autorin ein offenes Lebewesen für alles das, was auf ihn zukommt und er macht von allen Sinnen Gebrauch, um der Entwicklung gewachsen zu sein. In einem oft rätselhaften Stil, voller Metapher reisen Marica Bodrožić Figuren in der Erzählsammlung „Der Windsammler“ (2007) sowie im Gedichtband „Quittenstunden“ (2011) auf Inseln, oder vielleicht nehmen sie da Zuflucht in der Natur. Der Raum wird ausführlich beschrieben, öffnet sich in einem erzählerischen Prozess und wirkt befreiend. Dabei kommen die Figuren sich selbst näher. Der Wind ist in der Erzählsammlung als Schlüsselmetapher für positive Entwicklungen zu sehen. Er wirkt auf nachdenkliche Menschen und erfrischt ihre Erinnerung. Einer der Figuren meint, dass nur jemand, der als Schelle der Erinnerung lebt, das Gedächtnis von seiner Belastung befreien kann. Nur so gelingt jeder zu seinen Wurzeln (WS, ${ }^{25}$ 163). Bodrožić Protagonisten sind Inselbesucher, Windsammler, Wolken u.a. Die Erzählsammlung wurde von der Literaturkritik an mehreren Stellen als realitätsfern, voller Aberglauben und enervierend überfüllter Metapher kritisiert. Bodrožić entgegnet, dass sowohl das Wahrhafte als auch das Richtige in der Sprache unfassbar sei. Gerade die Spannung zwischen dem unumgänglichen Schreib- bzw. Erzählbedürfnis und der Erkenntnis der sprachlichen Unangemessenheit ist das, was den Sinn ihrer Kunstwerke bestimmt. ${ }^{26}$ Sie weiß die Sprache zu schätzen, denn die Geschichte wird erfahrbar in und durch die Sprache. ${ }^{27}$ In der Literatur sieht sie einen neuen Ansatz zur Erklärung der Geschichte. Interdisziplinarität und Methodenvielfalt sind zur Interpretation der Werke solcher Autoren notwendig. Dies ist eine Anforderung an die Germanistik. Nötig ist zudem eine Mentalitätsforschung

\footnotetext{
25 WS ist die hier gebrauchte Abkpürzung für das Werk „Windsammler“.

$26 \quad$ Vgl., Rock. S. 50.

27 Kazmierczak, Madlen 2012: Nation als Identitätskarte? Zur literarischen Auseinandersetzung mit ,Nation' und ,Geschichte' bei Marica Bodrožić und Melinda Nadj Abonji. In: Germanica. 51. S. $21-33$, hier: S. 27.
} 
im Laufe von Akkulturationsprozessen. ${ }^{28}$ Die Interpretation eines Kunstwerks ist nicht weniger wichtig als seine Schöpfung, und setzt neben fundierter Wissenschaft auch Sensibilität voraus.

In ihrem Aufsatz „Nirgendwo Liebe, aber Augustlicht“ (2009) schreibt Bodrožić über Jugoslawien: , [...] alle glauben, dass die Grenzen keine mehr sind, weil man sie nicht mehr sehen kann." (Nirgendwo, ${ }^{29}$ 116). In ihrem Aufsatz begründet sie, es gebe kein „Erinnnerungsschießen“ (Nirgendwo, 115). Sie spricht von „Hautgedächtnis.“ (Nirgendwo, 118), also von seelischen Wunden aus der Kindheit, die lebenslang ihre Spuren hinterlassen. Ist der „Windsammler“ mit Metapharn überfüllt, so setzt sie sich auseinander mit dem Grenzproblem in „Nirgendwo Liebe, aber Augustlicht“" auseinander und kommt zu dem Schluss: „Das Denken tut dann weh, so wie das Gehen weh tut, wenn man in den Muskeln Muskelkater hat.“ (Nirgendwo, 111f). Im ursprünglichen Vielvölkerstaat Jugoslawien ging es um ein spontanes unbewusstes uneingeschränktes und ungezwungenes Ineinandergehen und Zusammenwachsen, um eine gegenseitige impulsive Bereicherung. Genau dies verstand man unter Heimat, die Bodrožić zurückdenkend als hybrid beschreibt. So war wohl auch laut Bodrožić die serbischkroatische Sprache. Diese Feststellung trifft auf viele Länder Zentraleuropas zu: nämlich das Leben in unterschiedlichen Sprachräumen, das Verhältnis des Eigenen zum Fremden. ${ }^{30}$ Im lyrischen Gedichtband "Quittenstunden“ ist das lyrische Ich nicht zufrieden, denn das Innere sucht ununterbrochen Kontakt und Zusammenklang mit dem Äußeren. Es beklagt sich über Verlust, Schrecken, mangelnde Liebe u.a. und tastet enttäuscht nach schönen Erlebnissen ab. Manchmal will es sich verwandeln. Zwar erreicht das lyrische Ich nichts, aber es freut sich mindestens über seine Bewegungsfreiheit. Bodrožić Poesie hat prosaische Elemente und ihre Prosa hat poetische Elemente. Deshalb bescheinigt die Literaturkritik sie für ihren ersten Gedichtband Frische und

\footnotetext{
28 Bubia, Fawzi. 2007. S. 123. In: Akten des XI. Internationalen Germanistenkongresses Paris 2005 "Germanistik im Konflikt der Kulturen", 6, Migrations-, Emigrations- und Remigrationskulturen / betreut von Fawzi Boubia.

29 Nirgendwo wird als Abkürzung für den Aufsatz „Nirgendwo Liebe, aber Augustlicht“ benutzt.

$30 \quad$ Vgl., Kazmierczak, op.cit, S. 26.
} 
Eigensinn. 2008 meint Bodrožić, „Das Dazwischen der Sprachen, der Länder, der Echoräume, der Erinnerung [ist] eine unerschöpfliche Quelle, [...] Es ist [...] eine Erfahrung, die sich beim Schreiben einstellt. Das MehrweltgefühlD offenbart sich von selbst. ${ }^{\text {‘31 }}$ Welsch Bezeichnung „Patchwork-Identitat ${ }^{\star 32}$ trifft auf diese Aussage Bodrožićs zu. Sie fühlt sich als ewig Reisende. Auf ihren Reisen nimmt sie das Fremde nicht als fremd wahr. Ihre Identität beschreibt sie als das „unsichtbar auflaufende Prozessuale“ und durch jedes neue Buch kommt sie der Pluralität „dem Mehrweltgefühl“ “33 in sich näher. Dabei denkt Bodrožićs an Arthur Rimbaud, der im 19. Jh. schon von dem Menschen als Eisenbahnsammelpunkt für kulturelle Bewegungen sprach. Der Mensch wandert heimatlos und muss einsehen, dass er selber auch geschrieben wird.

Diese drei hier präsentierten Autoren widerspiegeln in den behandelten Werken an Hand ihrer Figuren verschiedene Stufen oder vielleicht nur verschiedene Perspektiven auf dem Weg der Identitätssuche, der Entflechtung und Verpflechtung. Während Zsuzsa Bánks Protagonisten einen anscheinend naiven, wenig reflektierten Weg anbahnen, leidet Saša Stanišićs Anti-Held an Zersplitterung. Bei Marica Bodrožić stößt man wiederholt auf Suchende, deren Sehnsucht nicht zu stillen ist. Keiner der Protagonisten kann sich ohne weiteres von der ursprünglichen Heimat - egal ob damit Nation gemeint ist, oder nur gemeinsame Geschichte einer Region - verabschieden. Die behandelten Werke machen es deutlich, dass in der Zeit des zusammenwachsenden Europas die Vielfalt anders zu verstehen ist als vor 1939. Gegen die Bestrebung der Vereinheitlichung Europas melden sich immer wieder Monokulturen, die gegen Assimilation und Homogenisierung kämpfen. Somit geht es um ein ständiges Hin und Her zwischen Verflechtung und Entflechtung. Kann die Theorie der Transkulturalität diese Problem lösen?

31 Pörksen, Uwe; Busch, Bernd (Hg.) 2008: Eingezogen in die Sprache, angekommen in der Literatur. Positionen des Schreibens in unserem Einwandrungsland. Göttingen: Wallstein. Hier S. 69f.

32 Welsch, Wolfgang 2012: Was ist eigentlich Transkulturalität? In: Lucyna Darowska/Claudia Machold (Hg.): Hochschule als transkultureller Raum? Beitrage zu Kultur, Bildung und Differenz. Bielefeld: transcript. 39-66, hier: S. 44.

33 Bodrožić , Marica: Die Sprachländer des Dazwischen. S. 67 - 75, hier S. 67. In: Uwe Pörksen; Busch, Bernd (Hg.) 2008: Eingezogen in die Sprache, angekommen in der Literatur. Positionen des Schreibens in unserem Einwandrungsland. Göttingen: Wallstein. 
Oder muss sie sich selber entfalten? In der wissenschaftlichen Literaturkritik wird der deutschen Literatur von Mittel- und Osteuropäiern - ganz gleich wie es klassifiziert wird - oftmals ein Inter- oder transkulturelles Potenzial zugesprochen. Da sie sich mit der Frage der persönlichen und kollektiven Identität auseinandersetzt, birgt diese Literatur sowohl Chancen als auch Schwierigkeiten in sich. Einerseits macht sie eindeutig, wie im Zeichen nationaler und ethnischer Determinationen Heimat, Familien und Beziehungen zerstört werden, andererseits enthält sie immer noch parallel die hierarchische Dichotomien vom Fremden und Eigenen. ${ }^{34}$ Folglich wird deutlich, dass die Vorstellung eines transkulturellen Weltmenschen, der sich als hybrider Charakter problemlos in einem dritten Raum bewegt und von dem Konzept Nation unberührt bleibt, vieles vereinfacht und unrealistisch ist. Denn jede Menschengruppe hat sowohl ihre spezifische Wahrnehmung als auch ihre Darstellungsformen, die kulturell vorgeprägt sind. Es ist deshalb von großer Bedeutung bei der Erforschung der Literatur Mittel- und Osteuropas zu untersuchen, inwiefern die Aspekte der „Interkulturalität“, der „Multikulturalität" und der „Transkulturalität“ bei der individuellen Selbstsuche und den Integrationsprozessen deutlich werden, inwiefern die Differenzen als zusammenhängende Differenzen erkennbar sind und ob es in dieser Literatur um eine - nach 1989 entstandene - „neue“ Transkulturalität geht, die von einer ,alten“ Transkulturalität klar zu unterscheiden sei, so Renata Makarska 2012. ${ }^{35}$ Transkulturalitatskonzepte sollen daraufhin in der Bellitristik eine Quelle für ihre Entfaltung suchen. ${ }^{36}$

\section{Literaturverzeichnis:}

ASSMANN, Aleida 2009: „Unbewältigte Erbschaften. Fakten und Fiktionen im zeitgenössischen Familienroman“, in : A. Kraft / M. Weißhaupt (Hrsg.), Generationen. Erfahrung - Erzählung - Identität, UVK. S. 49-69.

ASSMANN, Aleida 1999: Erinnerungsräume. Formen und Wandlungen des kulturellen Gedächtnisses. München: C.H.Beck.

$34 \quad$ Vgl., Kazmierczak: op.cit.

$35 \quad$ Vgl.: Makarska: op.cit. S. 257.

36 Kimmich, Dorothee 2012: Lob des »Nebeneinander «. Zur Kritik kulturalistischer Mythen bei Kafka und Wittgenstein. Darunter mehrere Aufsätze von den Autoren. In: Dorothee Kimmich $(\mathrm{Hg})$; Sigrid Karin Amos: Kulturen in Bewegung. Beiträge zur Theorie und Praxis der Transkulturalität. Bielefeld: transcript. S. 41- 68, hier S. 64. 
BÁNK, Zsuzsa $2013^{13}$ : Der Schwimmer. Frankfurt a.M.: Fischer.

BÁNK, Zsuzsa $2013^{12}$ : Die hellen Tage. Frankfurt a.M.: Fischer.

BODROŽIĆ , Marica 2011: Quittenstunden. Gedichte. Salzburg, Wien: Otto Müller Verlag.

BODROŽIĆ , Marica 2009: Nirgendwo Liebe, aber Augustlicht. In: Julia Franck, Marcel Beyer (Hg.): Grenzübergänge. Autoren aus Ost und West erinnern sich. Frankfurt a.M.: Fischer .

BODROŽIĆ , Marica 2009: Weder am Morgen noch in der Nacht. In: Julia Franck, Marcel Beyer: Grenzübergänge. Autoren aus Ost und West erinnern sich. Frankfurt a M: Fischer Verlag. S. $110-119$.

BODROŽIĆ , Marica 2007: Der Windsammler. Erzählungen. Frankfurt a.M.: Suhrkamp.

BORGOLTE, Michael; SCHNEIDMÜLLER, Bernd (Hg.) 2010: Hybride Kulturen im mittelalterlichen Europa. Berlin: Akademie Verlag GmbH.

BÜHLER-DIETRICH, Annette 2012: Verlusterfahrungen in den Romanen von Melinda Nadj Abonji und Saša Stanišić. In: Germanica 51. S. 35 - 46.

FINZI, Daniela 2013: Saša Stanišić: Wie der Soldat das Grammofon repariert. In: Unterwegs zum Andern? Literarische Er-Fahrungen der kriegerischen Auflösung Jugoslawiens aus deutschsprachiger Perspektive. Tübingen: francke Verlag. S. 235 - 252.

FISCH, Michael 2015: »Es erkenne mich die Welt, auf dass sie mir verzeihe«. Aufsätze zu Adelbert von Chamisso (1781-1838), Paul Ernst (1866-1933) und Hubert Fichte (1935-1986). Berlin: Weidler. (Beiträge zur transkulturellen Wissenschaft. Band 1.)

KAZMIERCZAK, Madlen 2012: Nation als Identitätskarte? Zur literarischen Auseinandersetzung mit ,Nation‘ und ,Geschichte‘ bei Marica Bodrožić und Melinda Nadj Abonji. In: Germanica. 51. S. 21-33.

KIMMICH, Dorothee 2012: Lob des »Nebeneinander«. Zur Kritik kulturalistischer Mythen bei Kafka und Wittgenstein. Darunter mehrere Aufsätze von den Autoren. In: Dorothee Kimmich (Hg); Sigrid Karin Amos: Kulturen in Bewegung. Beiträge zur Theorie und Praxis der Transkulturalität. Bielefeld: transcript. S. 41- 68.

KIMMICH, Dorothee; Schamma, Schahadat (Hg.) 2012: Kulturen in Bewegung. Beiträge zur Theorie und Praxis der Transkulturalität. Bielefeld: transcript.

MAĆEK, Amalija 2008: Balkanbilder bei Saša Stanišić und Catalin Dorian Florescu. In: Slavija Kabić, Goran Lovrić (Hg.): Mobilität und Kontakt. Deutsche Sprache, Literatur und Kultur in ihrer Beziehung zum südeuropäischen Raum. Kroatien: Verlag. S. 347 - 354.

MAKARSKA, Renata 2012: Kakanien der neuen Generation Zentraleuropa zwischen Transkulturalität und Differenz. In: Dorothee Kimmich (Hg); Sigrid Karin Amos: Kulturen in Bewegung. Beiträge zur Theorie und Praxis der Transkulturalität. Bielefeld: transcript. S. 235 -260 .

PöRKSEN, Uwe; Busch, Bernd (Hg.) 2008: Eingezogen in die Sprache, angekommen in der Literatur. Positionen des Schreibens in unserem Einwandrungsland. Göttingen: Wallstein.

PROPSZT, Eszeter 2007: Zur interdiskursiven Konstruktion ungarndeutscher Identiät in der ungarndeutschen Gegenwartsliteraur. Würzburg: Königshausen \& Neumann. S. 209. 
RoCK, Lene 2012: Überflüssige Anführungsstriche: Grenzen der Sprache in Terézia Moras Alle Tage \& Saša Stanišić' Wie der Soldat das Grammophon repariert. In: Germanica 51. S. 47-62.

SCHÜTTE, Andrea 2010: Ballistik. Grenzverhältnisse in Sašs Stanšićs „Wie der Soldat das Grammofon repariert“. In: Zeitschrift für deutsche Philologie. Werner Besch u.a. (Hg.). 129. Berlin: Erich Schmidt Verlag. S. 221 - 235.

STANIŠIĆ, Saša $2008^{3}$ : Wie der Soldat das Grammofon repariert. München: Verlagsgruppe Random House.

THUSWALDNER, Anton 2006: Die neuen Namen, in: Volltexte 28 (6). S. 5-7.

TRUCHLAR, Leo 2006: Zsuzsa Bánk. Der Schwimmer. In: Schwelle. Passage. Verwandlung. Ein Interpretationsentwurf. Wien: Lit Verlag GmbH. \& Co. KG. S. 335 - 337.

WELSCH, Wolfgang 2012: Was ist eigentlich Transkulturalität? In: Lucyna Darowska/Claudia Machold (Hg.): Hochschule als transkultureller Raum? Beitrage zu Kultur, Bildung und Differenz. Bielefeld: transcript. 39-66.

Akten des XI. Internationalen Germanistenkongresses Paris 2005 "Germanistik im Konflikt der Kulturen", 6, Migrations-, Emigrations- und Remigrationskulturen / betreut von Fawzi Boubia. 